\title{
MODELO CONSTRUTIVISTA PARA APOIAR O PROCESSO DE GESTÃO DA UNIVERSIDADE FEDERAL DE TOCANTINS
}

\section{RESUMO}

As instituições de ensino superior possuem impacto direto na promoção do crescimento econômico e desenvolvimento social do ambiente em que se inserem, seus resultados são esperados por governos, empresários e sociedade, surgindo assim a necessidade de ferramentas de gestão capazes de apoiar os processos decisórios. Neste contexto o presente estudo possui como indagação central Como um Modelo de Avaliação de Desempenho pode contribuir para o processo de gestão do Campus de Araguína da Universidade Federal do Tocantins (UFT)? Para responder ao questionamento tem-se como objetivo a estruturação de um modelo multicritério de avaliação de desempenho para apoiar a gestão do Campus Araguaína da Universidade Federal de Tocantins. O estudo de caso desenvolvido possui caráter exploratório de abordagem quali-quantitativa com coleta de dados primários e secundários. O instrumento de intervenção utilizado foi a Metodologia Multicritério de Apoio à Decisão Construtivista (MCDA-C), que atende os fundamentos da avaliação de desempenho como instrumento de apoio à decisão. Foram identificados 8 objetivos estratégicos, operacionalizados por 134 indicadores de desempenho, destes 33 encontram-se em desempenho comprometedor, evidenciando a necessidade de intervenção. O modelo facilitou a visualização desta situação e disponibilizou processo para a proposição de ações de melhorias, permitindo o monitoramento e o aperfeiçoamento da situação atual.

Palavras-Chave: Ensino Superior; Universidades; Avaliação do Desempenho; Metodologia MCDA-C.

\section{ABSTRACT}

Higher education institutions have a direct impact in promoting economic growth and social development. Specially of the environment on which they operate. Their results are expected by governments, businesses and society. For this reason, there is a need for management tools that can support decision-making processes. In this context, this present study main question is how a Performance Assessment Model can contribute to Araguina Campus management process of the Federal University of Tocantins (UFT)? To answer the question, the goal is to structure a performance evaluation multicriteria model to support the management of Araguaína campus of the Federal University of Tocantins. The developed case study has exploratory character with qualitative and quantitative approach, collecting primary and secondary data. The intervention instrument used was the Multicriteria Decision-Aid Methodology - Constructivist (MCDA-C). Methodology that meets the foundations of performance evaluation as a decision support tool. We identified 8 strategic objectives, operationalized by 134 performance indicators. Among these, 33 are in compromising performance, highlighting the need for intervention. The model facilitated the overview of this situation and provided a process for proposing improvement actions. Thus, enabling the monitoring and improvement of the current situation.

Key-words: Higher Education; Universities; Performance Evaluation; MCDA-C Methodology.

\author{
Leonardo Ensslin ${ }^{1}$ \\ Ademar Dutra ${ }^{2}$ \\ Renard Pereira Martins ${ }^{3}$ \\ Vinicius Dezem ${ }^{4}$
}

\footnotetext{
${ }^{1}$ Universidade do Sul de Santa Catarina, Brasil

E-mail: leonardoensslin@gmail.com

${ }^{2}$ Universidade do Sul de Santa Catarina, Brasil

E-mail: ademar.unisul@gmail.com

${ }^{3}$ Universidade do Sul de Santa Catarina, Brasil

E-mail: renaud@uft.edu.br

${ }^{4}$ Universidade do Sul de Santa Catarina, Brasil

E-mail: vinicius_dezem@hotmail.com
} 
Modelo Construtivista para Apoiar o Processo de Gestão da Universidade Federal de Tocantins

\section{INTRODUÇÃO}

As universidades são organizações sociais complexas, que passaram por diversas crises em sua história, para superar tais adversidades a formulação de estratégias e modelos de gestão para alcançar os resultados desejados por seus gestores. Observa-se que cada universidade possui peculiaridades que as distinguem das demais organizações, fator que torna o ambiente decisório conflituoso e repleto de alternativas (ANDRADE, 2002).

Não só as características das Instituições de Ensino Superior (IES) são complexas, seus sistemas de avaliação de desempenho são amplos, diversificados e em sua maioria genéricos (BUZZIGOLI et al., 2010; HONG, 2009), desta forma o presente estudo propõe a utilização de um instrumento de apoio á decisao, personalizado aos valores e preferências do decisor (gestor da universidade), acreditando que a avaliação de desempenho de IES seja o instrumento científico disponível para se apoiar a gestão de uma organização (ROSA et al., 2015; ENSSLIN et al., 2016).

Diante do contexto apresentado emerge a seguinte pergunta de pesquisa: Como um Modelo de Avaliação de Desempenho pode contribuir para o processo de gestão do Campus de Araguína da Universidade Federal do Tocantins (UFT)?

Com a finalidade de responder a esta pergunta o trabalho tem como objetivo geral a estruturação de um modelo multicritério de avaliação de desempenho que permita ao decisor apoiar o processo de gestão do Campus de Araguaína da UFT, fundamentado em uma visão Construtivista. Como objetivos específicos, destacam-se: (i) identificar as preocupações do decisor, quanto aos critérios para avaliar o desempenho da Universidade; (ii) mensurar ordinalmente e evidenciar o perfil de desempenho da situação atual (status quo) dos aspectos críticos para o sucesso do processo de gestão da UFT, segundo o entendimento do decisor; (iii) Propor ações de aperfeiçoamento aos indicadores considerados comprometedores.

A relevância deste trabalho se alicerça sobre um tema de pesquisa fundamentado na visão construtivista e, também na construção de um modelo de gestão que expande o conhecimento do decisor (gestor) a respeito do contexto específico que se pretende gerenciar.

Justifica-se a pesquisa por sua originalidade, importância e viabilidade (CASTRO, 1977), (i) original; em recente levantamento bibliográfico realizado não foi encontrado na literatura trabalho com o tema avaliação de desempenho de IES levando em conta a afiliação teórica construtivista, (ii) importante; este estudo permite expandir o entendimento do decisor em relação a organização, criar indicadores e a partir destes promover ações de melhoria, (iii) viável; por utilizar estrutura já existente na Universidade, em termos de pesquisadores e dados.
O presente trabalho é composto, além dessa Introdução, pelas seguintes seções: seção 2, com a Metodologia da Pesquisa; a seção 3, com a Análise dos Resultados; e a seção 4 com a apresentação das Considerações Finais.

\section{METODOLOGIA DA PESQUISA}

Para o presente estudo a metodologia de pesquisa aplicada será apresentada em duas etapas. A primeira refere-se ao enquadramento metodológico da pesquisa, e a segunda evidencia o instrumento de intervenção, Metodologia Multicritério de Apoio à Decisão Construtivista (MCDA-C), utilizado para a construção do modelo de avaliação de desempenho.

\subsection{Enquadramento Metodológico}

De acordo com Richardson (2010) o enquadramento metodológico engloba os seguintes fatores: (i) Natureza da pesquisa, o estudo é de caráter aplicado, na forma de estudo de caso, onde há a busca para entendimento e solução de um fenômeno real, obtida por meio da construção de um modelo de avaliação de desempenho para o processo decisório de uma IES. (YIN, 2015); (ii) Natureza do Objetivo, por expandir o conhecimento no decisor envolvido no processo, considera-se de natureza exploratória, busca ainda expandir o conhecimento sobre a área de atuação e partir disto obter um conjunto de critérios, que poderão ser transformados em indicadores de desempenho da gestão IES; (iii) Abordagem do problema, considera-se uma abordagem qualiquantitativa, de modo que os aspectos qualitativos ocorreram ao se identificar as preocupações do decisor, durante a fase de estruturação do modelo, em sequência ocorre a etapa quantitativa, ao desenvolver as escalas ordinais dos indicadores de desempenho (CRESWELL, 2010), o último fator a ser analisado é a (iv) Coleta de dados, que envolveu dados primários e secundários, (RICHARDSON, 2010). Os dados primários são oriundos das observações quando da coleta do perfil de status quo dos indicadores de desempenho. Os dados secundários são obtidos em decorrência de análises em documentos e relatórios de gestão.

\subsection{Metodologia Multicritério De Apoio À Decisão Construtivista - MCDA-C}

A metodologia foi escolhida por atender os requisitos para a construção do modelo, estes requisitos levados em consideração foram que: (i) - Os decisores desejam melhorar seu entendimento sobre o problema; (ii) - Os decisores desejam explicitar os fatores críticos para o sucesso de gestão do negócio; (iii) Os decisores desejam que as particularidades do ambiente sejam levadas em conta. Nos contextos gerenciais a objetividade, e a subjetividade são inerentes ao 
Modelo Construtivista para Apoiar o Processo de Gestão da Universidade Federal de Tocantins

processo decisório logo as situações que envolvem a tomada de decisão, devem ser analisadas a partir de ambos. (BANA E COSTA, 1993), (MICHELI; MARI, 2014). Desta forma a MCDA-C muda a ênfase de análise da ontologia (saber como é a realidade) para a epistemológica (expressar como a realidade é entendida, percebida) (MICHELI; MARI, 2014).

O processo da MCDA-C, também conhecido como abordagem Soft da Pesquisa Operacional, é composta por três fases, são elas:(i) Estruturação; (ii) Avaliação; (iii) Recomendações (ENSSLIN, et al., 2001).

i) A fase de Estruturação: tem por objetivo identificar os atores envolvidos no contexto, determinar as características do contexto decisório e modela-los de acordo com a percepção do gestor (MONTIBELLER et al., 2008).

ii) A fase de Avaliação: representa a parte quantitativa do trabalho, na qual as escalas ordinais são transformadas em escalas cardinais (mensuração) com a finalidade de permitir a construção do modelo de Apoio à Decisão (ENSSLIN, et al., 2001).

iii) A fase de Recomendações: é o processo de determinação de ações a serem seguidas para modificação do contexto e de acordo com as estratégias desenvolvidas, atingir os objetivos preferenciais do decisor (ENSSLIN, et al., 2001).

O presente estudo apresenta duas etapas da MCDA-C, a fase de estruturação e a fase de recomendações, possibilitando a identificação dos critérios julgados importantes pelos atores do contexto, a identificação de seu perfil de desempenho e a proposição de ações de melhoria.

\section{ESTUDO DE CASO}

Esta seção apresenta a estruturação do modelo de avaliação de desempenho para o processo de gestão da UFT, campus de Araguaína, embasado nos sistema de valores, interesses e preferencias dos decisores, levando em consideração os requisitos dos stakeholders (público de interesse) na percepção do decisor.

\subsection{A Uft: Campus De Araguaína}

A Universidade Federal do Tocantins é jovem, pelo pouco tempo de concepção tem um processo de gestão que ainda está em formação. Com 10 mil alunos, a instituição mantém 43 cursos de graduação oferecidos em sete campi, sendo eles: Palmas, Araguaína, Tocantinópolis, Miracema, Gurupi, Arraias e Porto Nacional. O foco desse trabalho está no Campus Universitário de Araguaína (TOCANTIS, 2008)

O Campus de Araguaína possui doze cursos de graduação: Matemática, Química, Geografia, Letras, Medicina Veterinária, Zootecnia, Química, Física, Biologia, Turismo, Logística e Cooperativismo. A unidade atende aproximadamente 2000 alunos e possui em seu quadro de servidores, uma média de 100 docentes e 100 técnicos administrativos.

\subsection{Fase De Estruturação}

A fase de estruturação da MCDA-C foi realizada em três etapas, sendo elas: a) identificação do problema ou contextualização: b) árvore dos pontos de vistas; c) e construção dos descritores (ENSSLIN et al., 2001; TASCA et al., 2012).

\subsubsection{Identificação do Contexto Decisório}

O processo de contextualização inicia com a identificação do subsistema de atores, estes são as pessoas inseridas no processo decisório, sendo classificados em decisores, intervenientes e facilitadores, ressalta-se a importância da valorização da subjetividade dos atores, ao prestigiar as peculiaridades internas e externas do contexto e motivações e preferências do gestor (ROY, 1993).

Os atores relacionados com a situação problemática a ser apresentada são os seguintes:

- Decisor: Diretor do Campus:

- Interveniente: Coordenadores de Cursos e Coordenadores administrativos;

- Agidos: Comunidade acadêmica e a comunidade em geral;

- Facilitador: Renard Pereira Martins.

Feita a identificação dos atores participantes do estudo de caso passou-se à descrição do macro ambiente no qual está inserido a situação problemática. $\mathrm{O}$ rótulo tem como objetivo apresentar o foco do trabalho ao mesmo tempo em que demonstra claramente o que deve ser atingido, sendo estabelecido como: "Construção de um Modelo de Apoio à Gestão da UFT - Campus de Araguaína"

Concluída as tarefas de identificação dos atores do processo decisório, descrição do ambiente, construção do rótulo e formulação do sumário finda-se a primeira etapa da fase de estruturação da MCDA-C e passa-se a construção da Árvore dos Pontos de Vista.

\subsection{2 Árvore dos Pontos de Vista}

O processo estruturado para a expansão do conhecimento necessário à construção da Árvore de Pontos de Vista é iniciado com a identificação dos Elementos Primários de Avaliação (EPAS). Os EPAS são as primeiras preocupações destacadas pelo decisor quando este é instigado a discorrer sobre a situação problemática (GRZEBIELUCKAS et al., 2011; ZAMCOPE et al., 2012).

Através da técnica de Brainstorming e com o apoio de um roteiro semiestruturado foi possível identificar no discurso do decisor aspectos que ele considerava importante para a gestão do contexto, onde o objetivo foi a maior quantidade possível e não a redundância das informações. 
Modelo Construtivista para Apoiar o Processo de Gestão da Universidade Federal de Tocantins

O passo seguinte à construção da Árvore de Pontos de Vista foi transformar os EPAS em conceitos. Esse processo foi feito a partir do aprofundamento do conhecimento acerca do respectivo EPA pelo decisor detalhando sua fonte de preocupação.

De acordo com Ensslin, et al., (2001) os conceitos são formados por um polo positivo (denominado objetivo subjacente) e por um polo oposto (denominado oposto psicológico). O objetivo subjacente representa o desempenho pretendido pelo decisor e o oposto psicológico é uma consequência que o decisor deseja evitar. O polo oposto e o objetivo subjacente são separados por (...) e quando lido significa "ao invés de". A interação entre facilitador e decisor proporcionou a construção de 216 conceitos e no quadro 1 são apresentados os conceitos para os 3 (três) primeiros EPAS.

Quadro 1. Conceitos dos Elementos Primários de Avaliação

\begin{tabular}{|c|l|}
\hline EPA & \multicolumn{1}{|c|}{ CONCEITO } \\
\hline \multirow{3}{*}{ 1- Saúde do Servidor } & $\begin{array}{l}\text { Assegurar que o ambiente de trabalho propicie condições físicas e } \\
\text { psicológicas para os servidores realizarem suas atividades...ter muitos } \\
\text { servidores afastados do trabalho para cuidar da própria saúde. }\end{array}$ \\
\hline 2- Educação do Servidor & $\begin{array}{l}\text { 2A- Assegurar que os servidores tenham capacitação e qualificação } \\
\text { adequada...ter atividades comprometidas por falta de capacitação e } \\
\text { qualificação dos servidores. }\end{array}$ \\
\cline { 2 - 2 } & $\begin{array}{l}\text { 2B-Assegurar que os servidores terceirizados tenham acesso à capacitação } \\
\text { periódica...ter comprometidas as atividades realizadas. }\end{array}$ \\
\hline \multirow{2}{*}{ 3- Conhecimento Técnico dos } & $\begin{array}{l}\text { Assegurar que os gestores tenham os conhecimentos técnicos necessários } \\
\text { para realização de suas atividades...ter atividades comprometidas por falta } \\
\text { de capacitação e qualificação dos servidores. }\end{array}$ \\
\hline
\end{tabular}

Fonte: Elaborado pelos Autores (2015).

A partir do entendimento inicial do decisor acrescido ao conhecimento gerado com a identificação dos EPAs e a construção dos conceitos o facilitador instiga ao decisor a estabelecer as grandes áreas de preocupação denotadas por candidatos a Pontos de
Vista Fundamentais-PVF, estes são percebidos pelo decisor como necessários e suficientes para a gestão do contexto em estudo. Deste processo top down emerge a primeira versão da Estrutura Hierárquica de Valor (ENSSLIN et al., 2016).

Figura 1. Árvore dos Ponto de Vistas Fundamentais

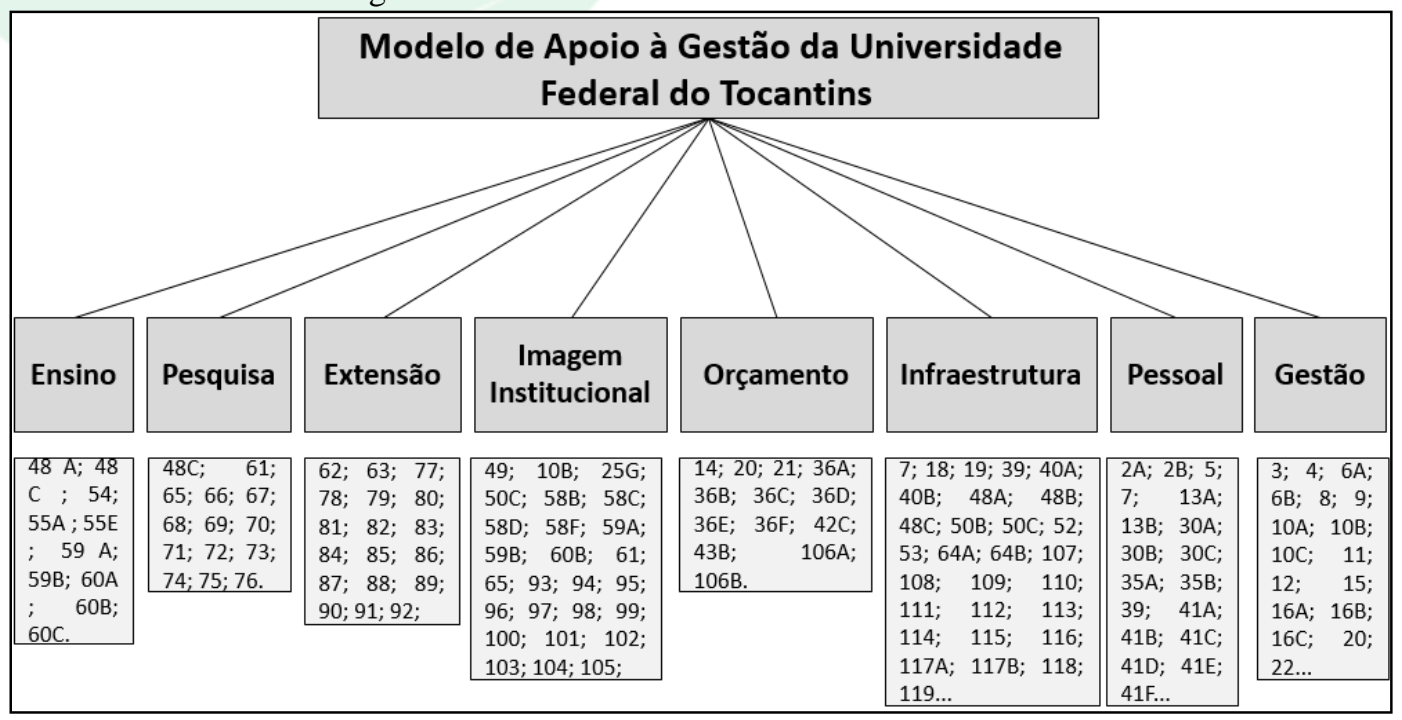

Fonte: Elaborado pelos Autores (2015).

De acordo com a Figura 1 o decisor elencou 08 (oito) áreas de preocupação e abaixo de cada uma delas foram relacionados os conceitos associados a área de preocupação, representados na estrutura por seus números identificadores. A construção dos conceitos, sua organização em áreas afins e elaboração da Árvore de Pontos de Vista finda esta etapa da fase de estruturação. 
Modelo Construtivista para Apoiar o Processo de Gestão da Universidade Federal de Tocantins

\subsubsection{Construção dos Descritores}

Para que os objetivos do decisor pudessem ser mensurados a MCDA-C vale-se de um processo denominado mapas de relações meios-fins ou mapas cognitivos (MONTIBELLER et al., 2008). A construção dos mapas cognitivos representa uma expansão do conhecimento do decisor a respeito de cada Área de Preocupação.
A construção dos mapas de relação meios-fim iniciou-se solicitando ao decisor para discorrer sobre cada conceito e sobre como a ideia subjacente ao mesmo poderia ser obtida (vínculos causais com outros conceitos), favorecendo a identificação das possíveis ações aptas a gerar efeitos no desempenho dos objetivos subjacentes (MONTIBELLER et al., 2008). A Figura 2 ilustra um dos mapas cognitivos para o $P V F$ - Processo Orçamentário:

Figura 2. Mapa Cognitivo do PVF- Processo Orçamentário

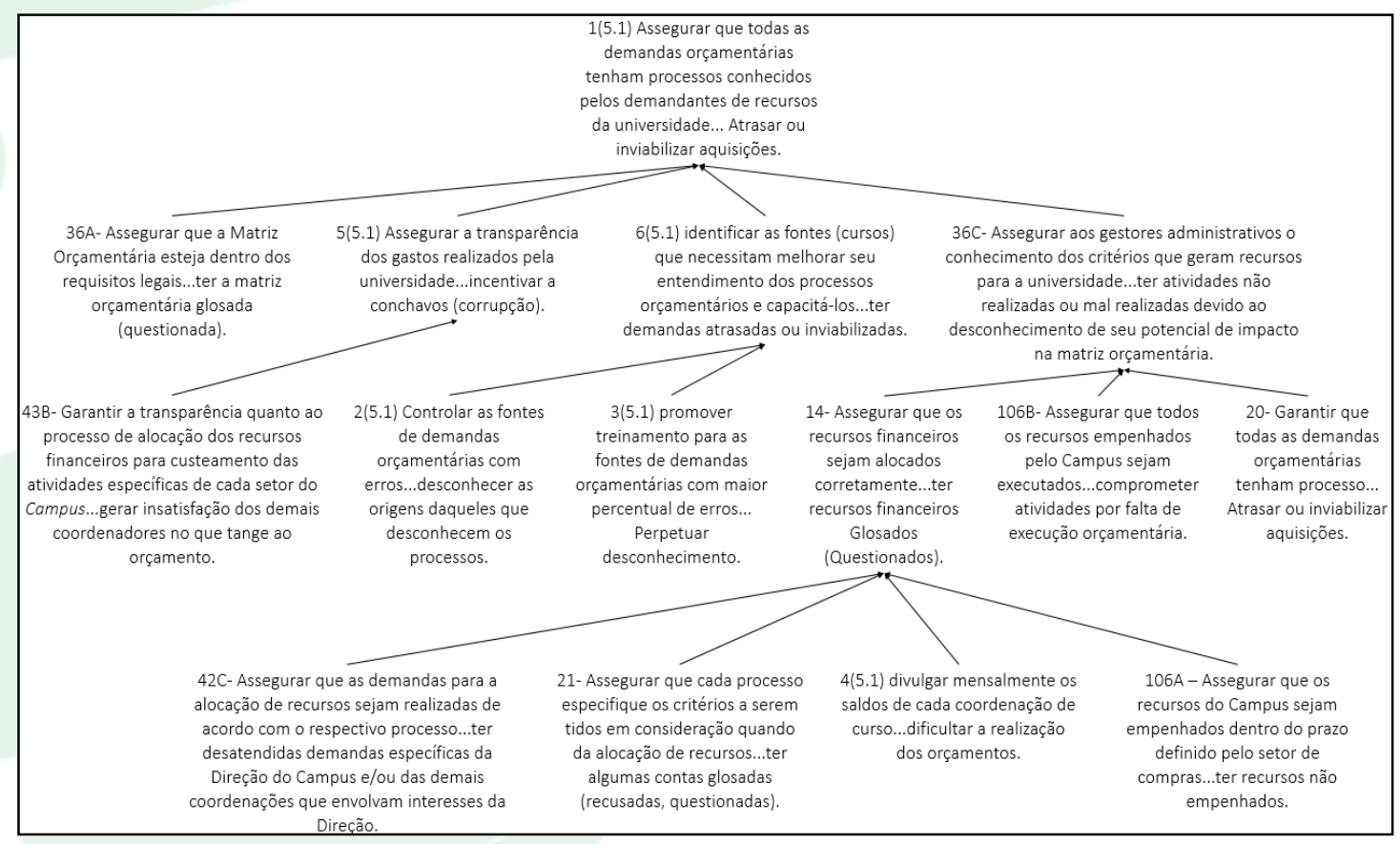

Fonte: Elaborado pelos Autores (2015).

Para todos os objetivos estratégicos da estrutura hierárquica top-down foram construídos os mapas de relação meios-fim. A MCDA-C, em seu processo de expansão do conhecimento sobre o contexto avaliado, sugere que as estruturas de relações causais sejam transformadas e transferidas para a Estrutura Hierárquica de Valor.

Com a finalização dos mapas cognitivos e construída a Estrutura Hierárquica de Valor-EHV passou-se a construção dos descritores, que representam indicadores de desempenho com finalidade de mensurar ordinalmente os aspectos ligados a cada PVF. Nessa etapa da estruturação foram construídos 134 (cento e trinta e quatro) descritores, estes devem evidenciar todos os possíveis impactos das ações potenciais de forma exaustiva, homogênea e não ambígua (TASCA et al., 2012).
Logo após definidos os níveis das escalas o decisor foi instigado a estipular para cada um dos descritores os níveis de referência e o perfil de desempenho (Status Quo) ordinal. Conforme apresentado por Tasca et al., (2012) os níveis de referência são denominados de "Nível BOM" e "Nível NEUTRO". O Nível BOM demarca o conjunto de ações que estão acima das expectativas do decisor. Por contraposição, o Nível NEUTRO delimita o conjunto de ações que se encontram em situação comprometedora. Todas as ações localizadas entre os Níveis BOM e NEUTRO se encontram de acordo com as expectativas do decisor (ENSSLIN, et al., 2001).

As faixas de desempenhos consideradas simultaneamente devem ser integradas conforme pode ser observado na apresentação da Figura 3. A EHV com os respectivos descritores associados ao PVF Formar Alunos é utilizada para ilustração. 
Modelo Construtivista para Apoiar o Processo de Gestão da Universidade Federal de Tocantins

Figura 3. Descritores da Área de Preocupação Ensino para o PVF formar alunos.

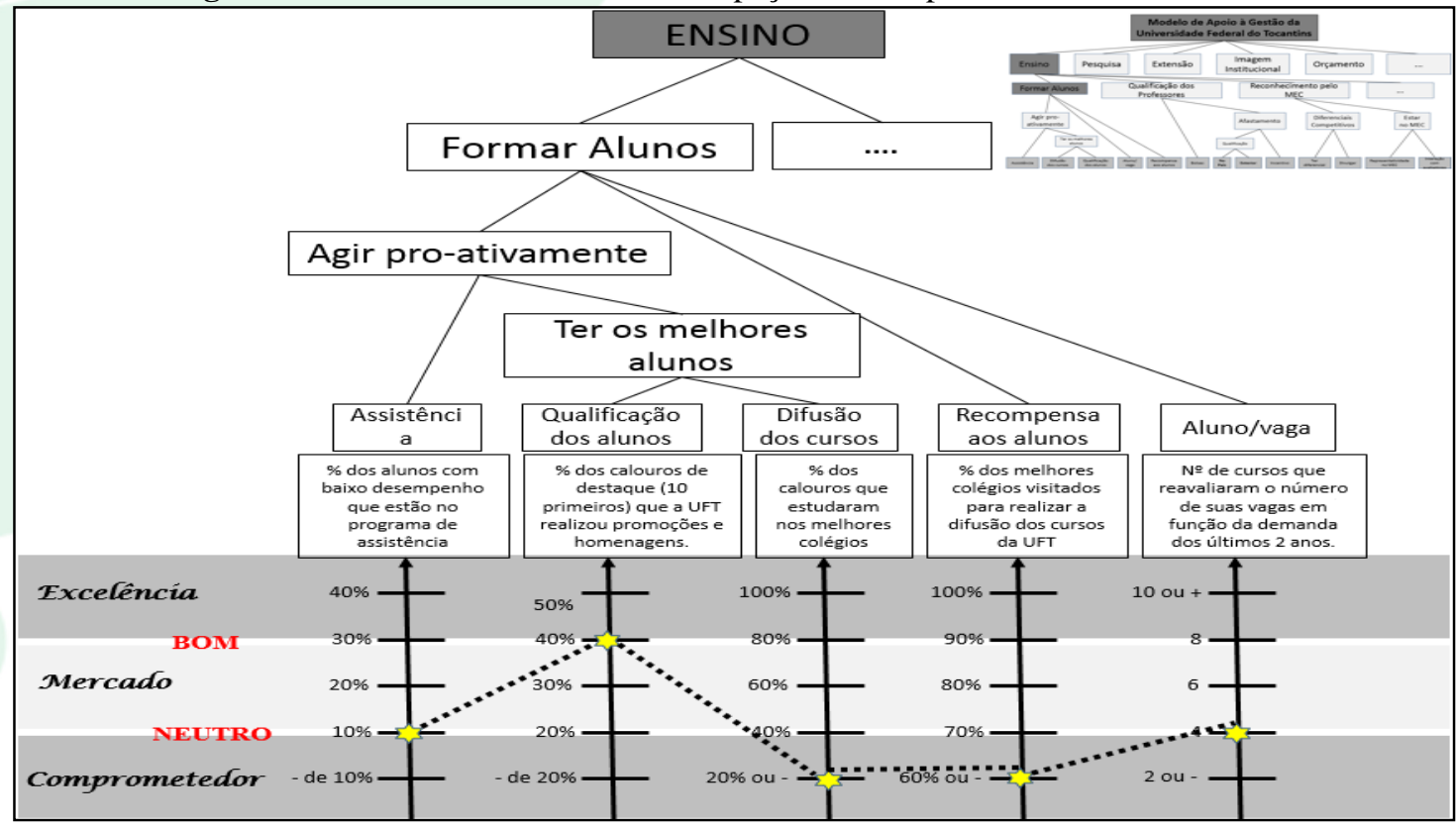

Fonte: Elaboração dos Autores (2015).

A definição dos níveis "BOM" e "NEUTRO" representados em cada descritor como a mesma preferência permite a caracterização dos descritores em três faixas de desempenho (LACERDA et al., 2014; ZAMCOPE; et al., 2012). Segundo os autores as faixas de desempenho são: a) Desempenho de excelência faixa de desempenho localizada acima do nível "BOM"; b) Desempenho de mercado ou competitivo - faixa de desempenho localizada entre o nível "BOM" e o nível "NEUTRO"; c) Desempenho comprometedor - faixa de desempenho localizada abaixo do nível "NEUTRO".

A partir da construção do descritor associado a cada escala pôde-se aferir a mensuração ordinal dos indicadores, sendo que 41 (quarenta e um) descritores estão em nível comprometedor, 60 (sessenta) descritores em nível de mercado e 33 (trinta e três) descritores em nível de excelência. As áreas de preocupação Infraestrutura, Gestão e Ensino se destacaram por apresentarem 22 (vinte e dois), 21 (vinte e um) e 20 (vinte) descritores respectivamente. Juntas, estas três áreas de preocupação representam $45 \%$ de todos os descritores do modelo de gestão. Os demais descritores (55\%) estão distribuídos pelas outras 5 (cinco) áreas de preocupação.

\subsubsection{Fase de recomendações}

A partir do modelo multicritério construído têmse a identificação de quais são as áreas estratégicas, as áreas táticas e as áreas operacionais. As áreas operacionais estão representadas pelas escalas dos descritores. As escalas são mensuradas ordinalmente e representam as preocupações que o decisor julga importante em cada um dos aspectos operacionais identificados (ENSSLIN, 2016).

Em seguida, para cada uma dessas escalas identificou-se em que nível de desempenho se encontra a situação atual. Esta informação permite ao decisor compreender, para cada descritor, se a performance está em nível comprometedor, normal ou de excelência (ENSSLIN, 2016).

Para cada um dos 41 dos descritores em nível de desempenho comprometedor foram geradas ações de melhorias, o modelo construído permitiu realizar um processo de aperfeiçoamento do contexto, podendo ser o planejamento de ações estratégicas para a gestão do Campus de Araguaína, possibilitando realizar o monitoramento e a identificação de ações de aperfeiçoamento para o planejamento de sua gestão.

\section{CONSIDERAÇÕES FINAIS}

O presente trabalho teve como situação problemática a maneira como avaliar o desempenho das universidades, e instigou a pesquisa sobre o tema gestão das Instituições de Ensino Superior e, de forma inédita associá-la à outra área de conhecimento: a Avaliação de Desempenho. Esta preocupação orientou o presente trabalho e fez surgir a pergunta de pesquisa: Como a Estruturação de um Modelo de Avaliação de Desempenho pode contribuir para o processo de gestão da Universidade Federal do Tocantins?

Foi estabelecido como objetivo geral estruturar um Modelo de Avaliação de Desempenho que permita apoiar o processo de gestão da Universidade Federal do Tocantins fundamentado na visão construtivista. $\mathrm{O}$ estudo de caso demonstrou a resolução da pergunta de 
Modelo Construtivista para Apoiar o Processo de Gestão da Universidade Federal de Tocantins

pesquisa ao proporcionar um modelo de gestão que transparece todos os objetivos estratégicos do decisor, assim como todos os meios a serem percorridos para alcançar os referidos objetivos. A busca para o atendimento do objetivo geral tornou necessário a desmembração deste nos seguintes objetivos específicos i) Identificar, mensurar ordinalmente e evidenciar o perfil da situação atual dos aspectos críticos do processo de gestão da UFT, segundo o entendimento do decisor; ii) Desenvolver processo que permita gerar ações de aperfeiçoamento aos desempenhos considerados comprometedores.

O primeiro objetivo específico foi alcançado pela realização do estudo de caso na UFT, por meio da utilização da MCDA-C, ainda na Fase de Estruturação. A Fase de Estruturação permitiu ao decisor identificar os aspectos específicos ao contexto e que deveriam ser levados em conta quando da construção do modelo de avaliação de desempenho. Posteriormente, o segundo objetivo específico foi atingido ao mensurar ordinalmente e evidenciar o perfil da situação atual dos aspectos julgados importantes pelo decisor, mostrando aqueles que lhe garantia destaque competitivo e também aqueles que lhe posicionava em nível comprometedor.

O terceiro objetivo específico foi alcançado na Fase de Recomendações da MCDA-C. Especificamente nessa fase desenvolveu-se um processo que permitiu ao decisor gerar ações de aperfeiçoamento aos desempenhos considerados comprometedores.

A importância do uso da MCDA-C é decorrente da necessidade do decisor responsável por formular as estratégias da instituição em saber o que está fazendo e

\section{REFERÊNCIAS}

Andrade, A. R. (2007). A universidade como organização complexa. Revista de Negócios, 7(3).

Bana E Costa, C. A. (1993). Três convicções fundamentais na prática do apoio à decisão. Pesquisa Operacional, 13(1), 9-20.

Buzzigoli, L., Giusti, A., \& Viviani, A. (2010). The evaluation of university departments. A case study for Firenze. International advances in economic research, 16(1), 24-38.

Castro, C. M. (1977). A prática da pesquisa. São Paulo: McGraw-Hill, 1977.

Creswell, J. W. (2010). Projeto de pesquisa métodos qualitativo, quantitativo e misto. In Projeto de pesquisa métodos qualitativo, quantitativo e misto. Artmed. quais as consequências das suas decisões no contexto de atuação.

Ainda sobre esse ponto de importância, reconhece-se que todo o conhecimento construído pelo decisor e explicitado no modelo de avalição será utilizado na gestão da IES ao qual pertence. Pode-se também afirmar que agora é possível exercitar na prática do dia-a-dia organizacional essa importante ferramenta de estratégia para o desempenho da instituição. A partir desse momento o conhecimento teórico se transforma em trabalho prático, sendo um instrumento laboral que amplia a visão estratégica de um gestor público, tão necessária para a complexidade encontrada nas instituições de ensino superior.

Por fim, é importante destacar como limitação desse trabalho a não realização da Fase de Avaliação da MCDA-C. Além disso, as ações apresentadas na Fase de Recomendações não foram implementadas para a averiguação dos resultados obtidos nesse contexto. Com isso, abre-se uma oportunidade de pesquisa que explore com dedicação a Fase de Avaliação da MCDAC e faça a aferição dos resultados da implementação das ações de melhoria.

Sugere-se para futuras pesquisas: i) a realização da fase de avaliação da MCDA-C; ii) aplicação prática da metodologia na Direção do Campus de Araguaína juntamente com a aferição dos referidos resultados; iii) criação de modelos de Avaliação de Desempenho, fundamentados na MCDA-C, para as diversas Coordenações de Cursos e Coordenações Administrativas do Campus de Araguaína; iv) a criação de modelos de Avaliação de Desempenho para outros Campus da Universidade Federal do Tocantins.

Ensslin, L., Dutra, A., Ensslin, S. R., Longaray, A. A. \& Dezem, V. (2016). Modelo Construtivista de apoio à Gestão Bancária. Espácios, 37(9), 12.

Ensslin, L., Neto, G. M., \& Noronha, S. M. (2001). Apoio à decisão: metodologias para estruturação de problemas e avaliação multicritério de alternativas. Insular.

Grzebieluckas, C., Buson, M. A., Queiroz, S. G., Ensslin, L., Ensslin, S., Nickel, E., \& Balbim, A. J. (2011). Instrumento para identificação das necessidades do consumidor no processo de desenvolvimento do design: Um estudo ilustrado com o projeto de um automóvel. Gestão \& Produção,18(2), 337-350.

Hong, D. R. (2009). A critical study on the university and academic assessment system in Korea. Inter-Asia Cultural Studies, 10(2), 292-302.

Lacerda, R. T. D. O., Ensslin, L., Ensslin, S. R., \& Dutra, A. (2014). A constructivist approach to manage 
Modelo Construtivista para Apoiar o Processo de Gestão da Universidade Federal de Tocantins

business process as a dynamic capability. Knowledge and Process Management, 21(1), 54-66.

Micheli, P., \& Mari, L. (2014). The theory and practice of performance measurement. Management accounting research, 25(2), 147-156.

Montibeller, G., Belton, V., Ackermann, F., \& Ensslin, L. (2008). Reasoning maps for decision aid: an integrated approach for problem-structuring and multicriteria evaluation. Journal of the Operational Research Society, 59(5), 575-589.

Richardson, R. J. (2010). Pesquisa social: métodos e técnicas.3 ed. São Paulo: Atlas.

Rosa, M. M., Ensslin, S. R., Petri, S. M., \& Ensslin, L. (2015). Avaliação de Desempenho de Políticas Públicas: Construção do Conhecimento com Base na Literatura Internacional. Revista Ibero-Americana de Estratégia,14(3), 110.
Roy, B. (1993). Decision science or decision-aid science?. European journal of operational research, 66(2), 184-203.

Tasca, J. E., Ensslin, L., \& Ensslin, S. R. (2012). A avaliação de programas de capacitação: um estudo de caso na administração pública. Revista de Administração Pública, 46(3), 647-675.

Tocantins, U. F. D.(2008) UFT em números.

Yin, R. K. (2015). Estudo de Caso-: Planejamento e Métodos. Bookman editora.

Zamcope, F. C., Ensslin, L., \& Ensslin, S. R. (2012). Desenvolvimento de um modelo para avaliar a sustentabilidade corporativa. Produção, Santa Catarina, 22(3), 477-489. 